\title{
Video Article \\ Using an Automated Cell Counter to Simplify Gene Expression Studies: siRNA Knockdown of IL-4 Dependent Gene Expression in Namalwa Cells
}

\author{
Adam M. McCoy ${ }^{1}$, Claudia Litterst ${ }^{1}$, Michelle L. Collins ${ }^{1}$, Luis A. Ugozzoli ${ }^{1}$ \\ ${ }^{1}$ Gene Expression Division, Bio-Rad Laboratories \\ Correspondence to: Michelle L. Collins at Michelle_Collins@Bio-Rad.com
}

URL: https://www.jove.com/video/1904

DOI: doi:10.3791/1904

Keywords: Cellular Biology, Issue 38, Cell Counting, Gene Silencing, siRNA, Namalwa Cells, IL4, Gene Expression, Electroporation, Real Time PCR

\section{Date Published: 4/14/2010}

Citation: McCoy, A.M., Litterst, C., Collins, M.L., Ugozzoli, L.A. Using an Automated Cell Counter to Simplify Gene Expression Studies: siRNA Knockdown of IL-4 Dependent Gene Expression in Namalwa Cells. J. Vis. Exp. (38), e1904, doi:10.3791/1904 (2010).

\section{Abstract}

The use of siRNA mediated gene knockdown is continuing to be an important tool in studies of gene expression. siRNA studies are being conducted not only to study the effects of downregulating single genes, but also to interrogate signaling pathways and other complex interaction networks. These pathway analyses require both the use of relevant cellular models and methods that cause less perturbation to the cellular physiology. Electroporation is increasingly being used as an effective way to introduce siRNA and other nucleic acids into difficult to transfect cell lines and primary cells without altering the signaling pathway under investigation. There are multiple critical steps to a successful siRNA experiment, and there are ways to simplify the work while improving the data quality at several experimental stages. To help you get started with your siRNA mediated gene knockdown project, we will demonstrate how to perform a pathway study complete from collecting and counting the cells prior to electroporation through post transfection real-time PCR gene expression analysis. The following study investigates the role of the transcriptional activator STAT6 in IL-4 dependent gene expression of CCL17 in a Burkitt lymphoma cell line (Namalwa). The techniques demonstrated are useful for a wide range of siRNA-based experiments on both adherent and suspension cells. We will also show how to streamline cell counting with the TC10 automated cell counter, how to electroporate multiple samples simultaneously using the MXcell electroporation system, and how to simultaneously assess RNA quality and quantity with the Experion automated electrophoresis system.

\section{Video Link}

The video component of this article can be found at https://www.jove.com/video/1904/

Protocol

\section{Preparing and counting the Namalwa cells}

1. Remove cells from culture flask and centrifuge the cells to pellet. Remove supernatant and resuspend cells in a known volume of PBS. The cells used in this protocol are suspension cells. If you are using adherent cells you will need to trypsinize before collecting.

2. To determine the number of live cells, stain an aliquot of the cell suspension by mixing it $1: 1$ with a $0.4 \%$ trypan blue solution and pipet $10 \mu l$ of the mixture onto a counting slide, and then insert the slide into the TC10 automated cell counter (Bio-Rad Laboratories, Inc).

3. The TC10 cell counter will autofocus to assure the most accurate count, and then proceed with counting. It automatically determines the density of live cells and total cells in the sample.

4. Select view image to display the cells on the viewscreen. Then select the dilution calculator to have the TC10 cell counter automatically calculate the amount of cell mixture that will be needed.

5. This experiment is using a total of three siRNAs: one control siRNA and two different siRNAs targeting the STAT6 gene, plus untransfected controls. This requires $3.6 \mathrm{ml}$ at a final concentration of $5 \times 10^{6}$ cells per $\mathrm{ml}$.

6. Enter the desired concentration and final volume values into the dilution calculator, and it calculates the volume of cell suspension needed based on the live cell count for that sample. Alternatively, perform the calculation manually. Then transfer the required volume of cell mixture for these experiments into a new tube.

7. Spin the cell mixtures to pellet the cells and remove all the PBS. Resuspend in $3.6 \mathrm{ml}$ of Gene Pulser electroporation buffer (BioRad Laboratories). Transfer $800 \mu$ laliquots into tubes containing the appropriate siRNAs and mix gently. The cells are now ready for electroporation.

\section{Electroporating the cells in $96-$ well Plates}

1. The 96-well electroporation plate allows the replicates for all four treatments to be electroporated together.

2. Transfer $150 \mu \mathrm{l}$ of the cell suspension into the appropriate wells of a 96 -well electroporation plate.

3. Insert the plate in the MXcell electroporation plate chamber and close the lid. 
4. Electroporate using settings optimized for the cell line and buffer being used. These cells are electroporated using an exponential decay wave with instrument settings of $250 \mathrm{~V}, 350 \mu \mathrm{F}$, and $1000 \Omega$ resistance.

5. After electroporation is complete, pipette up and down gently in each well to mix and then transfer cells to a tissue culture plate containing pre-warmed media. Namalwa cells are grown in RPMI (Life Technologies) supplemented with 7.5\% FCS and $1 \%$ pyruvate.

6. Control samples that have not been electroporated are taken from the remaining cell suspension and added to culture dishes of pre-warmed culture medium identically to the electroporated cells.

7. Incubate cells overnight at $37^{\circ} \mathrm{C}$ with $5 \% \mathrm{CO}_{2}$.

8. After $24 \mathrm{hr}$, one set of cells was induced with $10 \mathrm{ng} / \mathrm{ml}$ final concentration recombinant IL-4 (R\&D Systems). The second set of control cells only receive additional media. All cells are incubated an additional $24 \mathrm{hr}$ at $37^{\circ} \mathrm{C}$ and $5 \% \mathrm{CO}_{2}$.

\section{Extract and check RNA}

1. After a total of $48 \mathrm{hr}$ of growth, count the cells in each well, and transfer one aliquot from each well to a microfuge tube for RNA extraction, and freeze a second aliquot for western blotting or other protein analysis.

2. Centrifuge samples to pellet the cells, and then remove and discard the supernatant from each tube.

3. Proceed with the RNA extraction. We typically use the Aurum total RNA kit (Bio-Rad) according to the included protocol.

4. When monitoring siRNA mediated gene knockdown it is particularly important to verify RNA quality because degraded RNA will give misleading results. The $260 / 280$ ratio does not indicate RNA integrity therefore it is important to run the samples on a gel or microfluidic device to confirm RNA are not degraded.

5. To assess both quality and quantity in one step, these samples will be analyzed using the Experion automated electrophoresis system (BioRad).

6. First, heat samples. Then, prime, load, and vortex the Experion RNA chip. Insert the chip into the Experion electrophoresis station and start run.

7. After the run is complete, the Experion software will display results automatically in the form of an electropherogram, results table (that displays RNA concentration, ribosomal ratio, and the RNA quality indicator number), and virtual gel (which looks similar to a traditional RNA gel). High quality total RNA samples will typically have an RQI of 8-10.

\section{Real-time PCR}

1. After verifying the RNA quality, proceed with the cDNA reactions. This experiment used the iScript one-step RT-PCR mix (Bio-Rad) to combine cDNA synthesis and real-time PCR in one reaction.

2. Make a master mix containing all the reaction components other than the RNA templates for each primer set, and distribute those into the PCR plate.

3. After diluting the RNA to a uniform concentration, add the RNA samples to the appropriate wells. Each reaction is set up in triplicate.

4. Seal the plate, and place it into the CFX384 real-time PCR system (Bio-Rad). Select the appropriate protocol and begin the run.

5. Once the run is complete, gene expression is determined by comparing gene expression of the gene of interest normalized to a reference gene in cells electroporated with experimental siRNA to the same normalization of cells electroporated with a control siRNA.

\section{Analysis of siRNA knockdown}

1. The expression of CCL17 was monitored using quantitative real-time PCR to investigate the role of the transcriptional activator STAT6 in IL-4 dependent induction of CCL17. For this experiment, GAPDH was used as a reference gene. Alternate reference genes or multiple reference genes can be used in the same manner.

2. The CFX Manager software automatically normalized CCL17 expression to expression of the reference gene from the same sample and automatically compared the resulting normalized values to control treatments by simply selecting GAPDH as "reference" and the control treatment (control siRNA without IL-4 induction) as "control" in the experiment settings window of the CFX manager software.

3. Without IL-4 induction, transcription of CCL17 remained at low, constitutive levels comparable to the control treatment.

4. Cells induced with IL-4 and retaining a normal STAT6 pathway (i.e., control siRNA or untransfected control treatments) showed 8-10 fold induction of CCL17 expression.

5. Cells induced with IL-4 but with STAT6 expression inhibited by introduction of either STAT6 siRNA showed only about 2 fold higher CCL17 expression than the uninduced cells confirming the role of STAT6 in the IL-4 induced expression of CCL17. 


\section{Representative Results}

A)

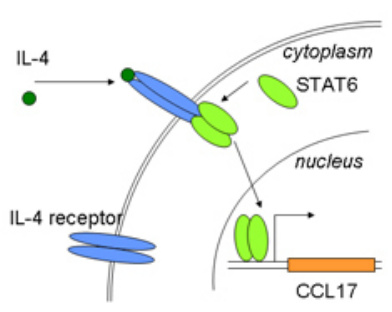

B)

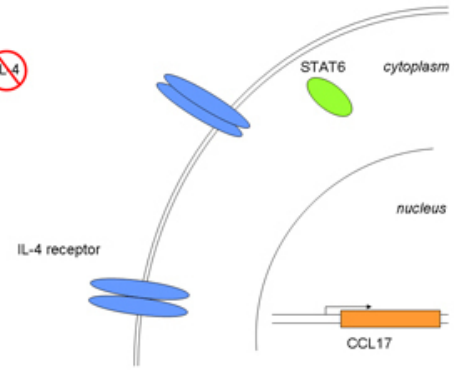

C)

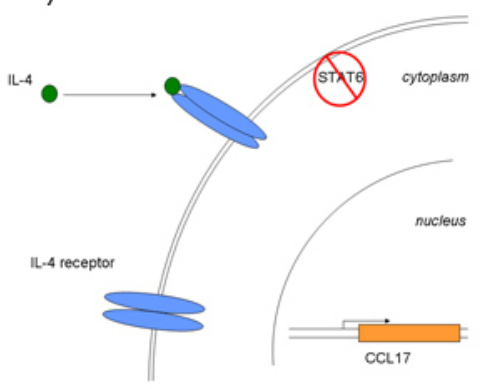

Figure 1. Overview of the IL-4 signaling pathway.

A) Binding of IL-4 to its receptor leads to dimerization and activation of STAT6. Activated STAT6 translocates to the nucleus and activates transcription of its target genes, such as CC17.

B) Without IL-4 induction, transcription of CCL17 will remain at low, constitutive levels.

C) After siRNA-mediated silencing of the STAT6 gene, treatment with IL4 should lead to little or no increase in CCL17 expression.

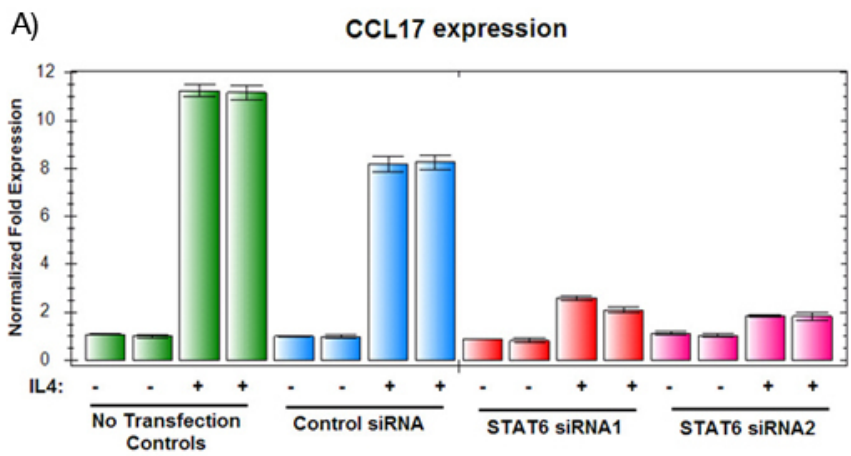

B)

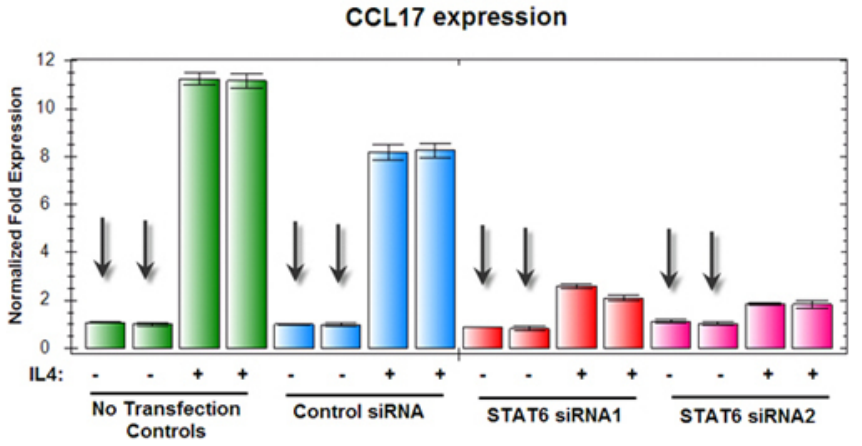

Figure 2. Induction of CCL17 by IL4 measured by different conditions by quantitative real time PCR.

A) Samples grown without IL-4 had a low constitutive level of expression regardless of gene expression. 
B) As shown in green and blue, control samples responded normally to treatment with IL-4; with an 8-10 fold induction of CCL17. However, cells transfected with siRNAs targeting STAT6, shown in red and pink, had diminished CCL17 expression in response to IL-4, supporting the idea that STAT6 plays a role in the IL-4 induced expression of CCL17.

\section{Discussion}

This article has demonstrated how to electroporate siRNAs into a difficult to transfect suspension cell line and how to follow gene expression in these cells. When doing this procedure it $\mathrm{s}$ important to remember to keep things as consistent as possible across all the samples.

\section{Disclosures}

The author is employed by Bio-Rad Laboratories that produces reagents and instruments used in this article. 